\title{
Correction to: BIOLUX P-III Passeo-18 Lux All-Comers Registry: 24-Month Results in Below-the-Knee Arteries
}

\author{
Gunnar Tepe $^{1} \cdot$ John Wang ${ }^{2} \cdot$ Jean-Marc Corpataux $^{3} \cdot$ Uei Pua $^{4} \cdot$ Christoph A. Binkert $^{5} \cdot$ \\ Matej Moscovic ${ }^{6} \cdot$ Reza Ghotbi $^{7} \cdot$ Koen Keirse $^{8} \cdot$ Donald Robertson' $^{9}$ \\ Marianne Brodmann ${ }^{10}$
}

Published online: 11 May 2021

(C) Springer Science+Business Media, LLC, part of Springer Nature and the Cardiovascular and Interventional Radiological Society of Europe (CIRSE) 2021

\section{Cardiovasc Intervent Radiol (2021) 44(1):10-18 https://doi.org/10.1007/s00270-020-02586-3}

The original version of this paper did not contain a list of BIOLUX P-III investigators. The purpose of this addendum is to acknowledge the contribution of all investigators who participated in the study.

Collaborators: BIOLUX P-III Global Registry Investigators: Marianne Brodmann, Thomas Zeller, JeanMarc Corpataux, Matej Moscovic, Gunnar Tepe, Koen Keirse, Giovanni Nano, Johannes B. Dahm, Johnny Kent Christensen, Reza Ghotbi, Christoph Binkert, Henrik Schröder, Denis Henroteaux, John Wang Chaw Chian, Eric
Rosset, Enrique Alejandre Lafont, Sabrina Houthoofd, Miguel Araujo, Shaiful Azmi Yahaya, Don Robertson, Martin Freund, Lonneke Yo, Uei Pua, Roxanne Wu, Frank Hammer, Michael Lichtenberg, Janne Korhonen, Della Schiava, Ralf Langhoff, Nicola Troisi, Secundino Llagostera, Jose-Maria Romero, Max Amor, Daniel Kretzschmar, Steven Kum, Patrice Mwipatayi, Albert J Smeets, Francisco Javier Serrano Hernando, Jost Philipp Schäfer, Gil Marques, Jos C. van den Berg, Ramesh Velu, Karlis Kupcs.

Publisher's Note Springer Nature remains neutral with regard to jurisdictional claims in published maps and institutional affiliations.

The original article can be found online at https://doi.org/10.1007/ s00270-020-02586-3.

Gunnar Tepe

gunnar.tepe@ro-med.de

1 Institut für Diagnostische und Interventionelle Radiologie, RoMed Klinikum Rosenheim, Pettenkoferstr. 10, 83022 Rosenheim, Germany

2 Department of Vascular Surgery, Singapore General Hospital, Singapore, Singapore

3 Department of Thoracic and Vascular Surgery, Lausanne University Hospital, Lausanne, Switzerland

4 Department of Diagnostic Radiology, Tan Tock Seng Hospital, Singapore, Singapore

5 Radiology Institute, Kantonsspital Winterthur, Winterthur, Switzerland

6 Department of Angiology, Institute of Cardiovascular Diseases, Kosice, Slovakia
7 Klinik für Gefäßchirurgie, Helios Klinikum München West, Munich, Germany

8 Department of Vascular Surgery, Regional Hospital Heilig Hart, Tienen, Belgium

9 Interventional Radiology, University Hospital Geelong, Geelong, Australia

10 Division of Angiology, Department of Internal Medicine, Medical University Graz, Graz, Austria 